\title{
Lily Poisoning in Domestic Cats
}

\author{
Welden Panziera', Claiton Ismael Schwertz'1, Luan Cleber Henker', Guilherme Konradt ${ }^{2}$, \\ Daniele Mariath Bassuino ${ }^{2}$, Rochana Rodrigues Fett ${ }^{3}$, David Driemeier ${ }^{1}$ \& Luciana Sonne ${ }^{1}$
}

\begin{abstract}
Background: Cases of plant intoxication in small animals are observed frequently in the domestic environment, mainly because most dogs and cats live in households and occasionally have access to streets and rural areas. Among such toxic agents, ornamental plants of the genus Lilium and Hemerocallis, which are potentially nephrotoxic to the feline species, are highlighted. Affected cats start presenting clinical signs 1-6 h after plant ingestion. Renal failure takes place in 12-72 $\mathrm{h}$, and death may occur in an interval ranging from three to seven days. The objective of this article is to describe the epidemiological, clinical and pathological findings of lily (Lilium sp.) poisoning in two cats.

Case: The aspects of lily poisoning in two cats are described (cat \#1 and cat \#2). Cat \#1 was a 3-year-old, mixed breed female cat, which presented a clinical history of anorexia, apathy, drooling, vomiting and polydipsia. Serum biochemical analysis revealed creatinine elevation $(21.2 \mathrm{mg} / \mathrm{dL})$, as well as hyperphosphatemia $(19 \mathrm{mg} / \mathrm{dL})$. Seventy-two $\mathrm{h}$ after the onset of clinical signs, renal failure progressed to anuria, followed by death. The second animal of this report (cat \#2) was a 2-year-old, mixed-breed male cat. The animal was found dead by the owner without displaying any previous clinical signs. Cats \#1 and \#2 ingested leaves of lily, which were present in their households as ornamental plants. At necropsy, the kidneys of both cats presented mild enlargement. Moderate perirenal edema was also noted. Cat \#1 showed morphologic extrarenal uremic lesions, characterized by ulcers in the oral mucosa and in the margin of the tongue ventral surface. Microscopic lesions observed in both cases were similar and compatible with acute toxic nephropathy. Histologically, severe epithelial cell degeneration and necrosis of proximal and distal convoluted tubules were noted. Other renal microscopic findings included hyaline and granular casts, tubule regeneration and occasional birefringent oxalate crystals. Cat \#1 also presented moderate white matter vacuolation in the telencephalon and cerebellum.

Discussion: The epidemiologic, clinical and pathological findings reported in the present study are similar to previous descriptions of lily poisoning in cats. Lily poisoning has been described in both males and females, without breed and age predisposition, similarly to what has been found in the present study. Kidney metabolite excretion, including the elimination of molecules such as creatinine, urea, and phosphorus is usually compromised in these cases, which was noted in cat \#1. The same animal showed extrarenal manifestations of renal failure, leading to a clinical presentation of uremic syndrome, which is not frequent in these intoxications. Animals intoxicated by lily usually die from renal failure and anuria. In most cases, lesions are restricted to the kidneys. In the reported cases, the microscopical lesions consisted of tubule epithelial cells degenerative changes and necrosis. Acute lily intoxication in cats must be differentiated from other conditions, such as intoxications due to aminoglycoside antibiotics, heavy metals, nonsteroidal anti-inflammatory drugs, antifungal agents, chemotherapeutic drugs, and ethylene glycol. The knowledge regarding the toxic potential of ornamental plants is fundamental in order to prevent such events of intoxication, as well as to reach the final diagnosis. Epidemiological, clinical and pathological findings were essential to conclude the final diagnosis.
\end{abstract}

Keywords: feline diseases, toxic plants, pathology, toxic nephropathy, uremia.

Descritores: doenças de felinos, plantas tóxicas, patologia, nefropatia tóxica, uremia. 


\section{INTRODUCTION}

Cases of plant intoxication in small animals are observed frequently in the domestic environment, mainly because most dogs and cats live in households, and occasionally have access to streets, parks and rural areas. Nonetheless, widespread ornamental plants present in gardens and used as house decoration are responsible for the majority of intoxications [1]. Among such toxic agents, ornamental plants of the genus Lilium and Hemerocallis, which are potentially nephrotoxic to the feline species, are highlighted. Some species of the referred genus, which may lead to intoxication, include the Eastern lily (Lilium longiflorum), the Peace lily (Spathiphyllum wallisii), the Tiger lily (Lilium tigrinum), the Japanese lily (Lilium speciosum) and Day lily (Hemerocallis spp.) [4,10].

Lily intoxication is characterized by kidney tubular necrosis in the feline species, and death is caused by acute renal failure. Affected cats may start presenting clinical signs 1-6 h after plant ingestion, which may include anorexia, apathy, vomiting, diarrhea, and drooling. Signs of renal failure usually occur from $12-72 \mathrm{~h}$ after lily ingestion, and are characterized by polydipsia, polyuria, anuria, and azotemia [2$4,6,8,10]$. The objective of this article is to describe the epidemiological, clinical and pathological findings of lily (Lilium sp.) poisoning in two cats.

\section{CASE}

The aspects of lily poisoning in two cats are described (cat \#1 and cat \#2). Cat \#1 was a 3-year-old, mixed breed female cat, which presented a clinical history of anorexia, apathy, drooling, vomiting and polydipsia. The cat owner informed that a few hours prior to the onset of clinical signs, the animal had ingested some leaves of lily, which was present in the house. Clinical examination revealed dehydration, hypothermia, and hypotension. Intravenous fluid therapy was performed. Abnormalities detected in serum biochemical analysis included creatinine elevation (21.2 $\mathrm{mg} / \mathrm{dL}$ \{reference range from 0.8-1.8 $\mathrm{mg} / \mathrm{dL}\}$ [5]), as well as hyperphosphatemia $(19 \mathrm{mg} / \mathrm{dL}$ \{reference range from 4.5-8.1 mg/dL\} [5]). Seventy-two h after the onset of clinical signs, renal failure progressed to anuria, followed by death. The second reported animal (cat \#2) was a 2-year-old, mixed-breed male cat that had free access to the outside environment. The animal was found dead by the owner without displaying any previous clinical signs. The owner reported that the cat was seen ingesting leaves of lily in the garden, corroborating to the clinical suspicion.

At the necropsy, cats \#1 and \#2 presented moderate dehydration, characterized by ocular globe retraction in the orbit. At necropsy, the kidneys of both cats presented mild enlargement as well as moderate perirenal edema. Cat \#1 showed morphologic extrarenal uremic lesions, which characterized the clinical presentation of uremic syndrome. The lesions consisted mainly of ulcerative stomatitis and glossitis, featured as ulcers in the oral mucosa (Figure 1) as well as bilateral and symmetrical ulcers and necrosis in the tongue ventral surface (Figure 2). Additional gross findings in both cats included moderate hydrothorax, pulmonary congestion and edema. During the necropsy, samples of several organs were collected, fixed in $10 \%$ buffered formalin, routinely processed for histology, and stained by hematoxylin and eosin.

Microscopic lesions observed in both cases were similar, and compatible with acute toxic nephropathy. Histologically, severe epithelial cell degeneration and necrosis of the proximal and distal convoluted tubules were noted (Figure 3). Degenerated cells were enlarged, and presented irregular and vacuolated cytoplasm. The necrotic epithelium was characterized by cells presenting granular or hypereosinophilic cytoplasm, with nuclear pyknosis and karyorrhexis, and sometimes nuclear absence. The lumen of numerous collecting ducts was filled with granular eosinophilic material (granular casts) or amorphous eosinophilic material (hyaline casts). Additionally, abundant amount of necrotic debris and occasional birefringent oxalate crystal were observed

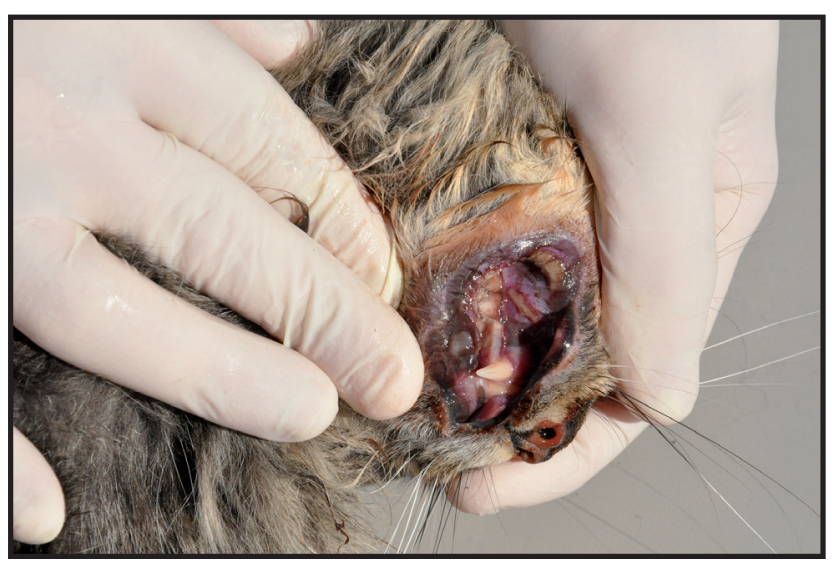

Figure 1. Spontaneous lily poisoning in domestic cats. Oral cavity. Multifocal areas of ulcerative stomatitis are observed in cat \#1. 


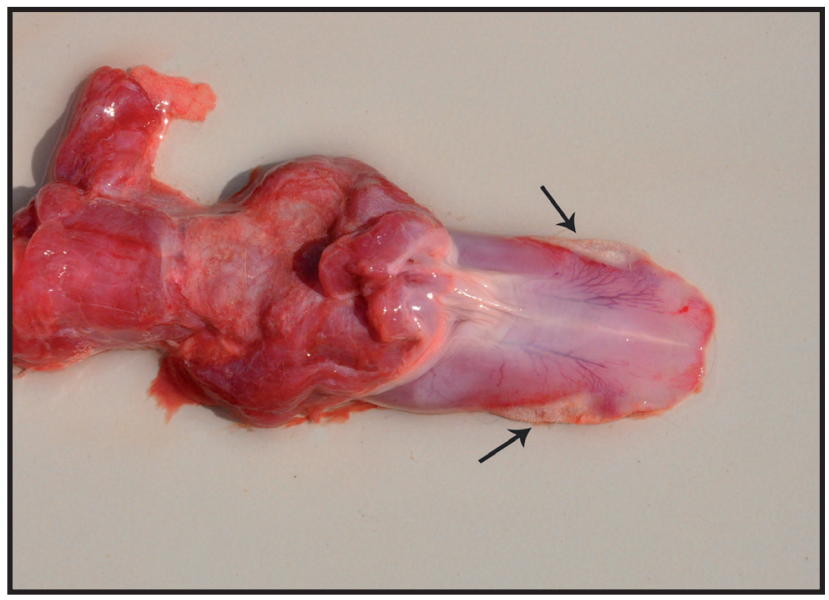

Figure 2. Spontaneous lily poisoning in domestic cats. Multifocal symmetric bilateral areas of ulceration and necrosis are observed in the margins of the tongue ventral surface in cat \#2 (arrows). These gross changes, along with the alterations noted in Figure 1, are consistent with extrarenal uremic lesions.

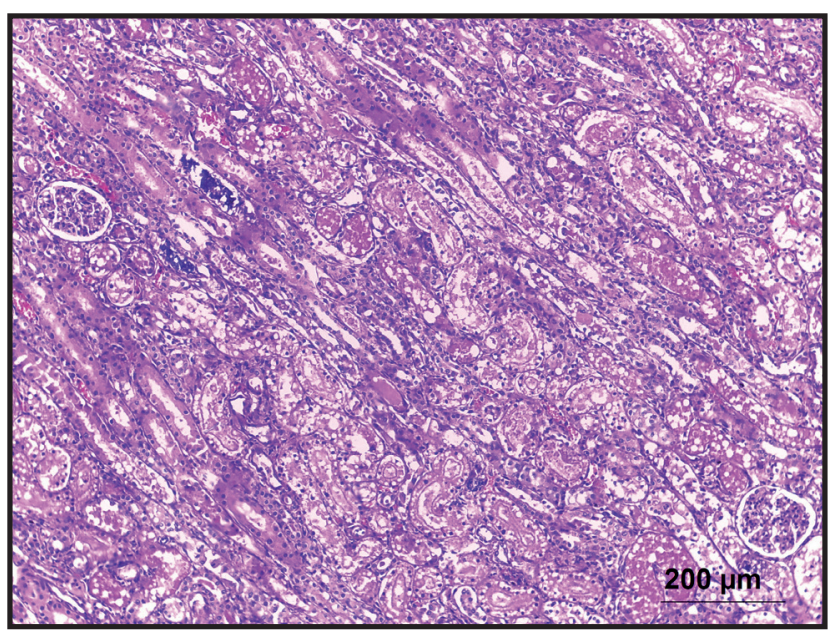

Figure 3. Spontaneous lily poisoning in domestic cats. Kidney. Severe epithelial cell degeneration and necrosis of the proximal and distal convoluted tubules are noted. In addition, granular and hyaline casts are seen in the lumen of numerous kidney tubules, associated with areas of mineralization [HE, x100].

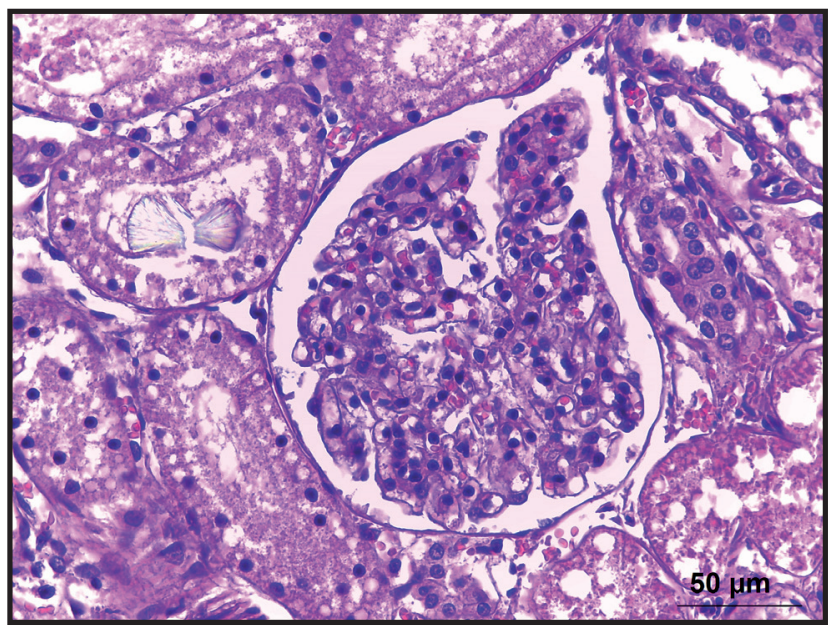

Figure 4. Spontaneous lily poisoning in domestic cats. Kidney. Birefringent oxalate crystal is observed in the lumen of a renal tubule [HE, $\mathrm{x} 400]$. inside proximal and distal convoluted tubules (Figure 4). Frequently, different degrees of tubular epithelial regeneration were also noted, as well as mild interstitial inflammatory infiltrate of lymphocytes, plasma cells, and macrophages.

Additional histological changes observed in cat \#1, which should be highlighted, included moderate white matter vacuolation of the cerebellum and telencephalon (occipital, parietal and frontal lobes); as well as bilateral focally extensive areas of necrosis and ulceration of the tongue epithelium, associated with mild fibrin deposition and mild inflammatory infiltrate of lymphocytes, plasma cells and neutrophils.

\section{DISCUSSION}

The epidemiological, clinical and pathological findings reported in the present study are similar to previous descriptions of lily poisoning in the feline species $[2-4,6,8,10]$. Cats are extremely susceptible to the intoxication by Lilium and Hemerocallis. The lack of knowledge regarding the toxic potential of such plants by the pet owners is a major predisposing factor [9]. Besides, lily intoxication risk is increased due to the widespread utilization of such plant in households, added to the fact that cats seem to present a peculiar attraction towards lily species [10]. It is suggested that some of these epidemiological factors may have predisposed to the intoxication event in the reported cases.

Lily poisoning has been observed in both males and females, without breed and age predisposition $[4,10]$, similarly to what has been found in the present study. All parts of the lily plant such as petals, stems, leaves, and pollen present toxic potential $[4,12]$. It was not possible to determine the ingested amount of lily in the reported cases; however, the consumption of small quantities, for instance, two leaves or a portion of a single flower may lead to death [4]. An experimental trial evaluating Oriental hybrid lily toxicity in cats demonstrated that doses of $5 \mathrm{~g} / \mathrm{kg}$ and $10 \mathrm{~g} / \mathrm{kg}$ of petals were lethal [12]. It is conjectured that cat \#2 of the present study may have ingested a greater amount of the toxic plant, due to the sudden death with no previous clinical signs.

Clinical signs are related to the nephrotoxic effects of lilies; even though the toxic principle and exact pathogenesis of renal toxicity are not known, the acute clinical presentation indicates a rapid absorption rate of the involved toxic molecule. Conversely, the 
nephrotoxic effects are not observed in dogs, even when great lily amounts are ingested [4]. The renal excretion of metabolites, such as creatinine, urea and phosphorus is compromised in such cases $[2,4,8,10]$. These changes were noted in cat \#1 of this report, which presented increased levels of creatinine and phosphorus. The association of azotemia, hyperphosphatemia and cylindruria indicates primary renal failure with tubular damage [2].

Affected cats may start presenting clinical signs 1-6 h after plant ingestion. Renal failure is established in 12-72 h, and death may occur in an interval ranging from three to seven days $[2,4,8,10]$. Similar aspects were noted in cat \#1 of the present report. Clinical signs of polydipsia, polyuria, and anuria indicate the acute renal failure. Additionally, the same animal displayed extrarenal manifestations of renal damage, leading to a clinical presentation of uremic syndrome. Uremic extrarenal changes, identified at the clinical examination or necropsy, are occasionally observed in cats intoxicated by lily [4], and are related to the longer survival time in the uremic phase of the disease. Therefore, the presence and severity of extrarenal lesions are greater in cases with a prolonged course of renal failure [3,7], as probably observed in cat \#1 of this study.

Animals intoxicated by lily usually die from anuric renal failure and total compromise of renal function [4]. In most cases, lesions are restricted to the kidneys $[2-4,6,8,10]$. In the reported cases, the renal microscopical lesions were compatible with acute nephrotoxic tubular necrosis, consisting of tubule epithelial cells degenerative changes and necrosis. Initial kidney lesions affect the proximal convoluted tubules, and with disease progression, distal convoluted tubules are also damaged. Ultrastructural changes include mitochondrial swelling in the proximal convoluted tubules epithelium, as well as megamitochondria formation, which may be the result of the enlargement of individual mitochondria as well as mitochondrial fusion. Additional ultrastructural changes described include piknotic nuclei in the tubule epithelial cells and fat infiltration [8]. Although not present in the reported cases, occasionally pancreatic lesions are associated with kidney lesions [6].

Acute lily intoxication in cats must de differentiated from other conditions which lead to toxic nephropathy and tubular necrosis. Among those, intoxications due to aminoglycoside antibiotics, heavy metals (for instance, lead, arsenic, and mercury), nonsteroidal anti-inflammatory drugs, antifungal agents (for example, amphotericin B), chemotherapeutic drugs (for instance, cisplatin), as well as ethylene glycol poisoning are highlighted [3,7]. In the present reported cases, the mentioned differential diagnoses were ruled out through the clinical history.

Toxin absorption may be considerably reduced, minimizing deleterious effects, when treatment is performed in the first hours of intoxication [11]. In the reported case, intravenous fluid therapy was performed in cat \#1, aiming to avoid the anuric phase of renal failure. Although the animal succumbed, fluid therapy plays a crucial role and must be conducted early in the disease progression, since dehydration, one of the main consequences of intoxication, may lead to death $[4,11]$.

The knowledge regarding the toxic potential of ornamental plants is fundamental in order to prevent such intoxications, as well as to reach the final diagnosis and establish the adequate treatment [11]. Furthermore, small animal clinicians and pathologists working with the feline species must consider lily intoxication as an important differential diagnosis in cats which present acute renal failure. Epidemiological, clinical and pathological findings were essential to conclude the final diagnosis.

Declaration of interest. The authors report no conflicts of interest. All authors approved the manuscript and its submission to the journal.

\section{REFERENCES}

1 Andrade S.F. 2011. Plantas tóxicas ornamentais. In: Nogueira R.M.B \& Andrade S.F. (Eds). Manual de Toxicologia Veterinária. São Paulo: Roca, pp.34-58.

2 Brady M.A \& Janovitz E.B. 2000. Nephrotoxicosis in a cat following ingestion of Asiatic hybrid lily (Lilium sp.). Journal of Veterinary Diagnostic Investigation. 12(6): 566-568.

3 Cianciolo R.E. \& Mohr F.C. 2016. Urinary system. In: Maxie M.G. (Ed). Jubb, Kennedy, and Palmer's Pathology of Domestic Animals. v.2. 6th edn. Philadelphia: Saunders Elsevier, pp.421-428.

4 Fitzgerald K.T. 2010. Lily toxicity in the cat. Topics in Companion Animal Medicine. 25(4): 213-217. 
5 Kaneko J.J., Harvey J.W. \& Bruss M.L. 1997. Clinical Biochemistry of Domestic Animals. 5th edn. San Diego: Academic Press, 932 p.

6 Langston C.E. 2002. Acute renal failure caused by lily ingestion in six cats. Journal of the American Veterinary Medical Association. 220(1): 49-52.

7 Newman S.J. 2013. O Sistema urinário. In: Zachary J.F. \& McGavin M.D. (Eds). Bases da Patologia em Veterinária. 5.ed. Rio de Janeiro: Mosby Elsevier, pp.592-662.

8 Rumbeiha W.K., Francis J.A., Fitzgerald S.D., Nair M.G., Holan K., Bugyei K.A. \& Simmons H. 2004. A comprehensive study of easter lily poisoning in cats. Journal of Veterinary Diagnostic Investigation. 16(6): 527-541.

9 Slater M.R. \& Gwaltney-Brant S. 2011. Exposure circumstances and outcomes of 48 households with 57 cats exposed to toxic lily species. Journal of the American Animal Hospital Association. 47(6): 386-390.

10 Souza T.M., Fighera R.A., Kommers G.D.\& Barros C.S.L. 2005. Poisoning by day lily (Hemerocallis sp.; Hemerocallidaceae) in Brazilian cats. In: Panter K.E., Wierenga T.L. \& Pfister J.A. (Eds). Poisonous plants: global research and solutions. Wallingford: CABI, pp.46-49.

11 Stumpf A.R.L., Gaspari R., Bertoletti B., Amaral A.S. \& Krause A. 2014. Intoxicação por lírio em um gato. Veterinária e Zootecnia. 21(4): 527-532.

12 Xia Z., Wan J., Chen Y., He Y. \& Yu J. 2013. Experimental oriental hybrid lilies (Lilium hybrids) poisoning in cats. Journal of Clinical Toxicology. 3(1): 1-4. 